\title{
Hydrogen peroxide production by ion irradiation of thin water ice films
}

\author{
O. Gomis ${ }^{1}$, G. Leto ${ }^{2}$, and G. Strazzulla ${ }^{2}$ \\ ${ }^{1}$ Dpt. Física Aplicada, Escuela Politécnica Superior de Alcoy (UPV), Placeta Ferrándiz Carbonell 2, 03801 Alcoy (Alicante), \\ Spain \\ 2 INAF - Osservatorio Astrofisico di Catania, via Santa Sofia 78, 95123 Catania, Italy
}

Received 23 January 2004 / Accepted 23 February 2004

\begin{abstract}
In this paper we present the results of new experiments on ion irradiation of water ice performed on thin films to study the synthesis of the hydrogen peroxide molecule and discuss the possibility of detecting it in icy mantles on interstellar grains. The used experimental technique has been in situ infrared spectroscopy. We have irradiated thin films (i.e. the ice thickness was smaller than the penetration depth of the used ion) with three different ions, namely $200 \mathrm{keV} \mathrm{of} \mathrm{H}^{+}$and $\mathrm{He}^{+}$and $400 \mathrm{keV}$ of $\mathrm{Ar}^{++}$. The experiments were carried out at temperatures of 16 and $77 \mathrm{~K}$. We have found that hydrogen peroxide is produced by all of the different ions at both temperatures. The detection of such a molecule has been possible from the study of its infrared feature centered at about $2850 \mathrm{~cm}^{-1}(3.5 \mu \mathrm{m})$. The obtained results also show that the produced $\mathrm{H}_{2} \mathrm{O}_{2} / \mathrm{H}_{2} \mathrm{O}(\%)$ ratio is greater for the heaviest ion $\left(\sim 6 \%\right.$ for the case of $\left.\mathrm{Ar}^{++}\right)$and that $\mathrm{H}^{+}$is the ion that produces the smallest quantity $(\sim 1 \%)$. These upper limits in the production of hydrogen peroxide constrain the quantity of $\mathrm{H}_{2} \mathrm{O}_{2}$ that can be formed after bombardment by cosmic particles on icy mantles of grains in the interstellar medium.
\end{abstract}

Key words. astrochemistry - methods: laboratory - techniques: spectroscopic - ISM: molecules - ISM: dust, extinction molecular processes

\section{Introduction}

Water is the dominant ice present in the Universe. It can be found in the Solar System as a constituent of the surfaces of some planets or satellites, of the trans-Neptunian objects, of some planetary rings (e.g., Saturn's rings), and also as part of the nucleus of the comets (see e.g., Schmitt et al. 1998). For a long time frozen water has been identified as the dominant component of ice mantles of dust grains in the interstellar medium (ISM) (Willner et al. 1982; Smith et al. 1989).

In dense interstellar and circumstellar environments absorption features attributed to other molecules in solid state have also been found by ground-based, air-borne and space observations (Ehrenfreund \& Schutte 2000). These ices are embedded in polar $\left(\mathrm{H}_{2} \mathrm{O}\right.$-rich) matrices and in non-polar (CO-rich) matrices depending on the local $\mathrm{H} / \mathrm{H}_{2}$ ratio present in the particular scenario (Pendleton \& Chiar 1997; Sandford et al. 1997; Whittet \& Tielens 1997; Ehrenfreund \& Fraser 2003). Among the solid state species that have been reported are $\mathrm{CO}, \mathrm{CO}_{2}, \mathrm{OCS}, \mathrm{H}_{2} \mathrm{CO}, \mathrm{HCOOH}, \mathrm{CH}_{4}, \mathrm{CH}_{3} \mathrm{OH}, \mathrm{NH}_{3}$, $\mathrm{OCN}^{-}$, and $\mathrm{NH}_{4}^{+}$(Boogert \& Ehrenfreund 2004).

However, the number of securely identified molecules is much lower than that predicted by theoretical models. In fact, chemical models that consider ion-molecule gas phase reactions and/or grain-surface reactions predict the existence of a

Send offprint requests to: $\mathrm{O}$. Gomis, e-mail: osgohi@fis.upv.es number of species in the solid state much greater than those detected up to now (Tielens \& Hagen 1982; d'Hendecourt et al. 1985; Hasegawa et al. 1992; Hasegawa \& Herbst 1993; Shalabiea \& Greenberg 1994). As an example, the hydrogen peroxide molecule $\left(\mathrm{H}_{2} \mathrm{O}_{2}\right)$ has been predicted to be produced on grain surfaces by hydrogenation of molecular oxygen (Tielens \& Hagen 1982; Hasegawa et al. 1992; Hasegawa \& Herbst 1993). The calculations made by those authors predict $\mathrm{H}_{2} \mathrm{O}_{2}$ abundances not greater than $5 \%$ with respect to $\mathrm{H}_{2} \mathrm{O}$.

Solid molecules in the interstellar and circumstellar medium can also undergo chemical reactions driven by energy released from energetic charged particle irradiation and/or UV photolysis. When an energetic ion or a UV photon collides with the ice, part of the deposited energy destroys molecular bonds in the target, producing radicals that can then react to synthesize new molecules. To study some of the effects induced by the radiation, and to identify part of the newly produced molecules, laboratory experiments on ice irradiation are carried out under physical conditions as close as possible to the astrophysical ones (e.g. Johnson 1998; Strazzulla 1998; Moore et al. 2001; Baragiola et al. 2003).

In a recent paper, Gomis et al. (2004) presented results on ion implantation in water ice (i.e. with a film thickness greater than the penetration depth of the used ion) to study the formation of the hydrogen peroxide molecule. The experiments were carried out using five different ions, namely $\mathrm{H}^{+}, \mathrm{C}^{+}, \mathrm{N}^{+}, \mathrm{O}^{+}$ 
and $\mathrm{Ar}^{+}$at two different temperatures, 16 and $77 \mathrm{~K}$. They have shown that hydrogen peroxide has been produced by all of the ions at both temperatures. It also has been shown that the quantity of $\mathrm{H}_{2} \mathrm{O}_{2}$ produced is much greater for the heaviest ions. In particular, the maximum quantity of hydrogen peroxide formed has been about $4 \%$ for $\mathrm{O}^{+}$at $77 \mathrm{~K}$. The obtained results have been applied to the Galilean satellite Europa in which hydrogen peroxide has been observed (Carlson et al. 1999).

Another recent work on irradiation of water ice to study and characterize the hydrogen peroxide molecule produced by radiolysis is that accomplished by Moore \& Hudson (2000). They have carried out experiments on irradiation (with $800 \mathrm{keV} \mathrm{H}^{+}$) of thin water ice and mixtures (film thickness $\sim 5 \mu \mathrm{m}$ ), at temperatures of 16 and $80 \mathrm{~K}$. Their results show that $\mathrm{H}_{2} \mathrm{O}_{2}$ is produced after irradiation of water at $16 \mathrm{~K}$, and the presence of oxidants such as $\mathrm{O}_{2}$ and $\mathrm{CO}_{2}$ in the icy mixture enhances the production of $\mathrm{H}_{2} \mathrm{O}_{2}$ by radiolysis.

In this paper we present new experiments on ion irradiation of water ice that complement those carried out by Gomis et al. (2004). In this case we have irradiated thin films of water ice (i.e. with a film thickness smaller than the penetration depth of the used ion) at temperatures of 16 and $77 \mathrm{~K}$. The used ions have been $200 \mathrm{keV}$ of $\mathrm{H}^{+}$and $\mathrm{He}^{+}$, and $400 \mathrm{keV}$ of $\mathrm{Ar}^{++}$. The experimental results are discussed in view of their relevance to the icy mantles present on grains in the interstellar and/or circumstellar medium. We have obtained an upper limit for the quantity of icy hydrogen peroxide that can be found on the icy grain mantles. This paper is structured as follows. In Sect. 2 we describe the experimental equipment. Our results are presented in Sect. 3 and the discussion in Sect. 4. We conclude with the astrophysical applications which are discussed in Sect. 5.

\section{Experimental apparatus}

The infrared spectra here shown have been obtained by infrared absorption spectroscopy performed in a high-vacuum chamber $\left(P<10^{-7} \mathrm{mbar}\right)$ faced, through IR-transparent windows, to a FTIR spectrophotometer (Bruker Equinox 55). Pre-prepared gases (or mixtures) are admitted into the chamber by a needle valve and subsequently are accreted as ices on a silicon crystal substrate. The substrate is put in thermal contact with a closed-cycle helium cryostat whose temperature can be varied between 10 and $300 \mathrm{~K}$. The vacuum chamber is interfaced to an ion implanter (200 kV; Danfysik 1080-200) from which ions with energy up to $200 \mathrm{keV}$ (400 keV for double ionization) can be obtained. The ion beam produces a spot greater than the area probed by the infrared beam. To avoid macroscopic heating of the target the current density is maintained in the range of $100 \mathrm{nA} \mathrm{cm}^{-2}$ to a few $\mu \mathrm{A} \mathrm{cm}^{-2}$. The integrated ion flux (fluence in ions $\mathrm{cm}^{-2}$ ) is directly related to the ion current measured during irradiation. The substrate plane is placed at an angle of 45 degrees with the IR beam and the ion beam so that spectra can be easily taken in situ, even during irradiation, without tilting the sample. Spectra can also be taken after warm up at selected temperatures in the range $10-300 \mathrm{~K}$. All the spectra shown in the following have been taken with a resolution of $1 \mathrm{~cm}^{-1}$ and a sampling of $0.25 \mathrm{~cm}^{-1}$. In our experiments we have used films with a thickness of $\sim 0.5 \mu \mathrm{m}$. More details

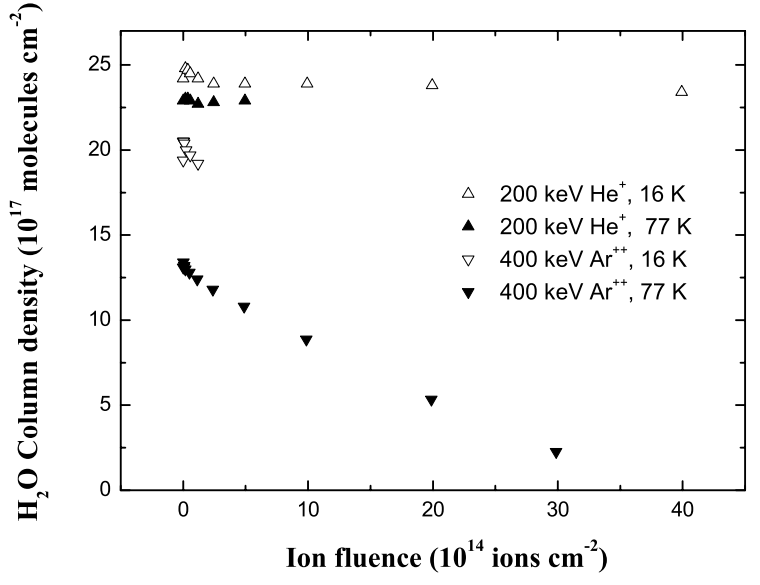

Fig. 1. Column density $\left(\times 10^{17}\right.$ molecules $\left.\mathrm{cm}^{-2}\right)$ of the water ice stretching band as a function of the ion fluence for $200 \mathrm{keV} \mathrm{He} e^{+}$and $400 \mathrm{keV} \mathrm{Ar}{ }^{++}$at temperatures of 16 and $77 \mathrm{~K}$.

on the experimental setup have been recently given (Strazzulla et al. 2001).

To calculate the column density of a given species $N$ (molecules $\mathrm{cm}^{-2}$ ) the following equation is used:

$N=\frac{\int \tau_{\nu} \mathrm{d} v}{A}$,

where $\int \tau_{v} \mathrm{~d} v\left(\mathrm{~cm}^{-1}\right)$ is the integrated area measured on the optical depth scale and $A$ is the integrated band absorbance $\left(\mathrm{cm} \mathrm{molecule}{ }^{-1}\right)$. The integrated area has been calculated by removing the underlying continuum using only two fixed points.

Water ice films have been obtained depositing $\mathrm{H}_{2} \mathrm{O}$ vapour onto a silicon substrate at a rate of about $2 \AA \mathrm{s}^{-1}$. Deposition times have varied between 30 and $60 \mathrm{~min}$ approximately. In this way we have obtained a film thickness that ranges between 3600 and $7200 \AA$.

\section{Results}

\section{1. $\mathrm{H}_{2} \mathrm{O}$ destruction}

Figure 1 shows the water ice column density (molecules $\mathrm{cm}^{-2}$ ), measured from the integrated intensity of the $3275 \mathrm{~cm}^{-1}$ $(3.053 \mu \mathrm{m})$ band, as a function of ion $\left(200 \mathrm{keV} \mathrm{He}^{+}\right.$, $400 \mathrm{keV} \mathrm{Ar}^{++}$) fluence at temperatures of 16 and $77 \mathrm{~K}$. An integrated absorbance $A\left(\mathrm{H}_{2} \mathrm{O}\right)=2.0 \times 10^{-16} \mathrm{~cm} \mathrm{molecule}^{-1}$ (Allamandola et al. 1988) has been used to evaluate the column density. All data sets plotted in Fig. 1 exhibit no initial $\mathrm{H}_{2} \mathrm{O}$ loss at low irradiation fluences (less than $1 \times 10^{14}$ ions cm$^{-2}$ ). This apparent increase in the amount of water ice is due to the variation of the band strength induced by ion irradiation. Leto \& Baratta (2003) have recently shown that the band strength of water ice undergoes a $10 \%$ increase already at low ion fluences. This behavior at low fluences is probably due to the competition between the increase of the band strength and the destruction process of the water ice layer. However, in this work we prefer to use the integrated absorbance of an unirradiated ice because the new value has not been measured at $77 \mathrm{~K}$. In addition, even at $10 \mathrm{~K}$ an increase of only about $10 \%$ has been observed but a new value has not been evaluated. 


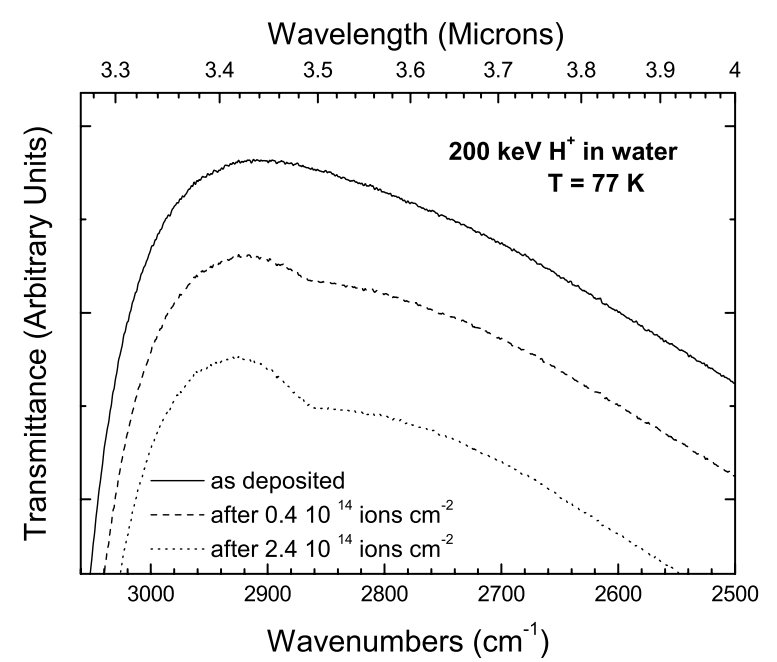

Fig. 2. IR transmission spectra of $\mathrm{H}_{2} \mathrm{O}$ ice as deposited (solid line) and after irradiation with $200 \mathrm{keV} \mathrm{H}^{+}$at $77 \mathrm{~K}$ with fluences of $0.4 \times$ $10^{14}$ and $2.4 \times 10^{14}$ ions $\mathrm{cm}^{-2}$ (dashed and dotted lines respectively). The appearance of an absorption band centered at $2852 \mathrm{~cm}^{-1}$ testifies to the formation of hydrogen peroxide molecules. The spectra after irradiation are shifted downwards for clarity.

From Fig. 1 the $\mathrm{H}_{2} \mathrm{O}$ destruction yield (molecules ion ${ }^{-1}$ ) can be obtained by means of a linear fit. We have been able to calculate this quantity only for $\mathrm{He}^{+}$at $16 \mathrm{~K}$ and for $\mathrm{Ar}^{++}$ at $77 \mathrm{~K}$ obtaining values of $26 \pm 8$ and $370 \pm 10$ molecules ion $^{-1}$ respectively. The case of $200 \mathrm{keV} \mathrm{H}^{+}$is not shown since for this ion we have obtained a slight increase in the $\mathrm{H}_{2} \mathrm{O}$ column density attributed to the increase in the band strength. We must stress that the destruction yields given here are not equivalent to the sputtering yields. The latter represent the number of molecules lost from the target. The destruction yields shown here include both the molecules sputtered away from the target and the molecules which are transformed into some new species. As a result, destruction yields shown above should be greater than the corresponding sputtering yields. In a recent paper, Baragiola et al. (2003) presented a review on sputtering of water ice. Looking, as an example, at their Fig. 3 it can be seen that the sputtering yield for $200 \mathrm{keV} \mathrm{He}^{+}$, in the range $20-77 \mathrm{~K}$, is lower than 20 molecules ion $^{-1}$. In addition, from their Fig. 5 one expects a value of the sputtering yield for $400 \mathrm{keV} \mathrm{Ar}^{++}$ lower than 300 molecules ion $^{-1}$. Thus, the results here reported are in agreement with the values obtained by those authors.

\section{2. $\mathrm{H}_{2} \mathrm{O}_{2}$ band}

Figure 2 shows the IR transmission spectra, in the $3060-2500 \mathrm{~cm}^{-1}$ range, of water ice deposited at $77 \mathrm{~K}$ and after irradiation with $200 \mathrm{keV}$ of $\mathrm{H}^{+}$at two different fluences (0.4 and $2.4 \times 10^{14}$ ions $\mathrm{cm}^{-2}$ respectively). In this particular case the water ice sample is about $0.6 \mu \mathrm{m}$ thick. The appearance of an absorption feature near the long wavelength wing of the $3 \mu \mathrm{m} \mathrm{H} \mathrm{H}_{2} \mathrm{O}$ band testifies to the formation of a new species. This new band, produced after irradiation, is attributed to the $\left(v_{2}+v_{6}\right) / 2 v_{6} \mathrm{OH}$ bending feature of the hydrogen peroxide molecule (Giguere \& Harvey 1959; Miller \& Horning 1961). The spectra show that the feature is centered at about
Table 1. Hydrogen peroxide destruction and formation cross-sections calculated by using Eqs. (3) and (4) and maximum $\mathrm{H}_{2} \mathrm{O}_{2} / \mathrm{H}_{2} \mathrm{O}(\%)$ ratio obtained from Figs. 4 and 5.

\begin{tabular}{lllll}
\hline \hline Ion & $\begin{array}{l}\text { Temp. } \\
(\mathrm{K})\end{array}$ & \multicolumn{2}{c}{ Cross-sections } \\
destruction $\left(\sigma_{\mathrm{d}}\right)$ & $\begin{array}{l}\text { formation }\left(\sigma_{\mathrm{f}}\right) \\
\times 10^{-15} \mathrm{~cm}^{2}\end{array}$ & $\begin{array}{l}\text { Maximum } \\
\mathrm{H}_{2} \mathrm{O}_{2} / \mathrm{H}_{2} \mathrm{O}(\%)\end{array}$ \\
\hline $\mathrm{H}^{+}$ & 16 & $9.0 \pm 1.2$ & $0.099 \pm 0.017$ & 1.2 \\
$\mathrm{H}^{+}$ & 77 & $16 \pm 5$ & $0.056 \pm 0.021$ & 0.4 \\
$\mathrm{He}^{+}$ & 16 & $13.0 \pm 1.0$ & $0.29 \pm 0.02$ & 2.4 \\
$\mathrm{He}^{+}$ & 77 & $30 \pm 5$ & $0.38 \pm 0.05$ & 1.3 \\
$\mathrm{Ar}^{++}$ & 16 & $3.8 \pm 0.4$ & $0.115 \pm 0.008$ & 6 \\
$\mathrm{Ar}^{++} 77$ & $9.5 \pm 2.0$ & $0.26 \pm 0.05$ & 3.0 \\
\hline
\end{tabular}

$2850 \mathrm{~cm}^{-1}(3.5 \mu \mathrm{m})$ which is in agreement with previously published work (Moore \& Hudson 2000).

\section{3. $\mathrm{H}_{2} \mathrm{O}_{2}$ production}

In Fig. 3 the percentage abundance of hydrogen peroxide is reported as a function of the ion fluence at two different temperatures, i.e., 16 and $77 \mathrm{~K}$, for the case of $\mathrm{H}^{+}, \mathrm{He}^{+}$and $\mathrm{Ar}^{++}$. This $\mathrm{H}_{2} \mathrm{O}_{2} / \mathrm{H}_{2} \mathrm{O}$ ratio, calculated in percentage and by number of molecules, has been obtained using Eq. (1). To obtain the column density for the $\mathrm{H}_{2} \mathrm{O}_{2}$ ice, we have used a value of the integrated band absorbance taken from the literature. The used value has been $A\left(\mathrm{H}_{2} \mathrm{O}_{2}\right)=2.7 \times 10^{-17} \mathrm{~cm} \mathrm{molecule}^{-1}$ (Moore $\&$ Hudson 2000).

The quantity of hydrogen peroxide produced is greater at $16 \mathrm{~K}$ than at $77 \mathrm{~K}$ for $\mathrm{H}^{+}$and $\mathrm{He}^{+}$ions, and for $\mathrm{Ar}^{++}$it is the same at both temperatures. This result is in agreement with the work by Moore \& Hudson (2000) who irradiated water ice films with $800 \mathrm{keV}$ of $\mathrm{H}^{+}$. They found lower $\mathrm{H}_{2} \mathrm{O}_{2}$ yields at $80 \mathrm{~K}$ compared to $16 \mathrm{~K}$. They suggested than the lower yield at the higher temperature could be traced to a change in the balance between $\mathrm{H}_{2} \mathrm{O}_{2}$ formation and destruction, which our Table 1 confirms and quantifies. They also proposed several reaction mechanisms to give a reason for this unexpected observation (see Moore \& Hudson 2000; Hudson \& Moore 2001, for further details).

In Figs. 4 and 5 we plotted the $\mathrm{H}_{2} \mathrm{O}_{2} / \mathrm{H}_{2} \mathrm{O}(\%)$ ratio, by number of molecules, as a function of the deposited energy (dose). This quantity is given in $\mathrm{eV}$ per small molecule (16 amu). In this case we use dose (eV/16 amu) instead of ion fluence (ions $\mathrm{cm}^{-2}$ ) because the former is a better way to compare the results obtained with different ions and by different authors. The doses have been calculated using SRIM2003 (Ziegler et al. 1985), which is a program that simulates the interaction of ions with matter and calculates the energy released to the sample.

Figures 4 and 5 demonstrate that the maximum amount of $\mathrm{H}_{2} \mathrm{O}_{2}$ produced at 16 and $77 \mathrm{~K}$ increases as the atomic number of the incoming ion increases. Thus $\mathrm{Ar}^{++}$is the ion that produces the greatest, and $\mathrm{H}^{+}$the ion that produces the smallest quantity. This result is in agreement with the previous work of Gomis et al. (2004) with thick film experiments, i.e., implantation of ions in samples having a thickness larger than 

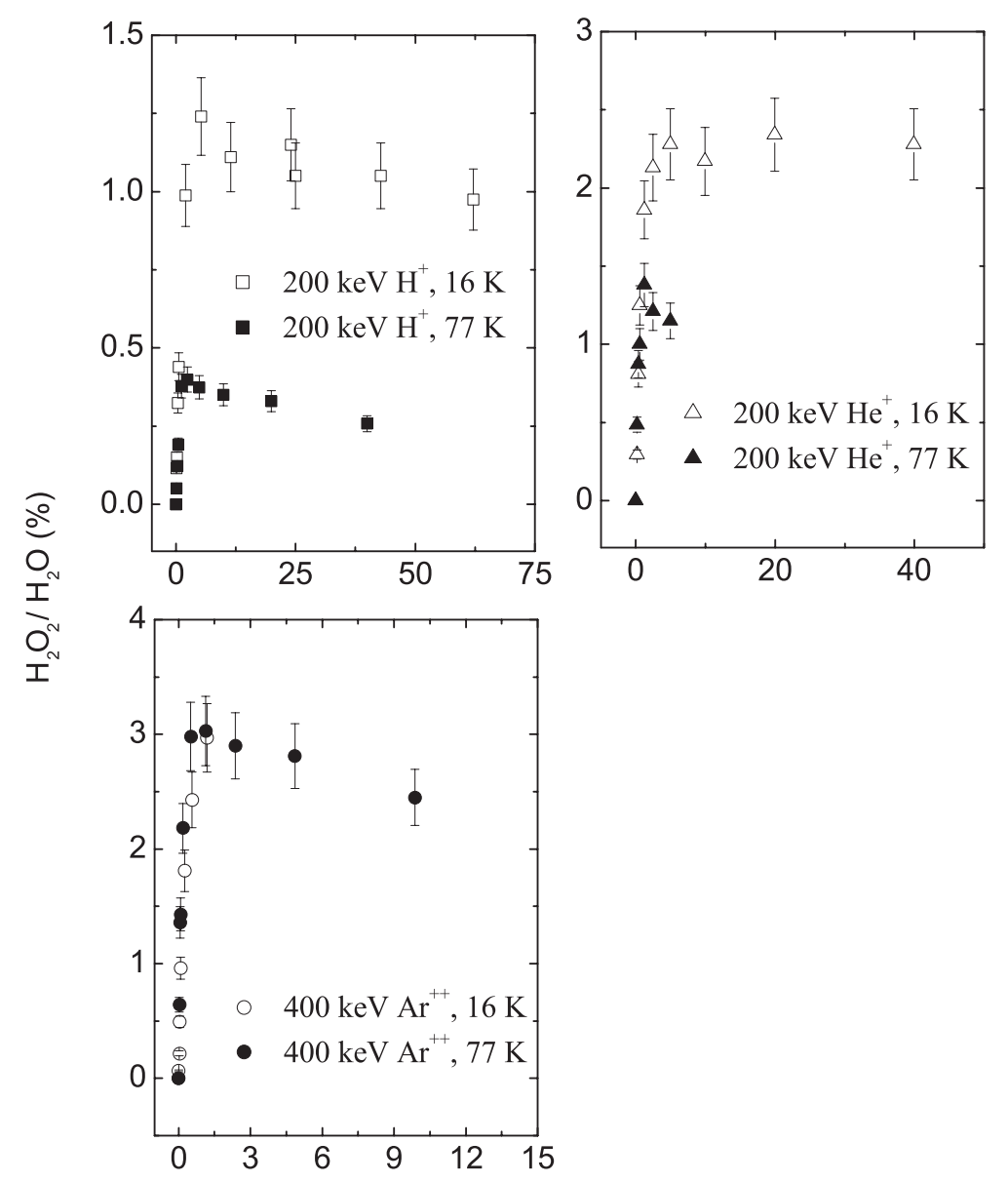

$$
\text { Ion fluence }\left(10^{14} \text { ions } \mathrm{cm}^{-2}\right)
$$

Fig. 3. Percentage of $\mathrm{H}_{2} \mathrm{O}_{2} / \mathrm{H}_{2} \mathrm{O}$ in number of molecules for each of the used ions at temperatures of 16 and $77 \mathrm{~K}$. The ratio is plotted as a function of the ion fluence (ions $\mathrm{cm}^{-2}$ ).

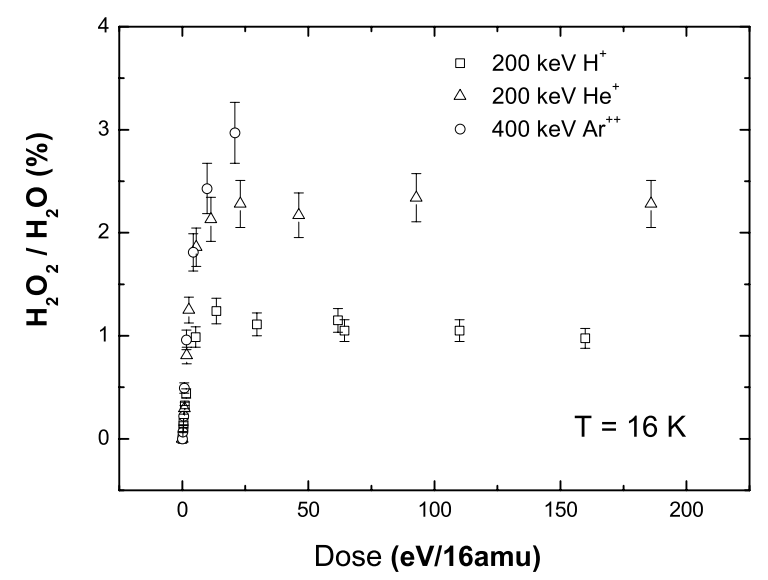

Fig. 4. $\mathrm{H}_{2} \mathrm{O}_{2} / \mathrm{H}_{2} \mathrm{O}(\%)$ production (in number of molecules) after irradiation of thin films of water ice at a temperature of $16 \mathrm{~K}$. The used ions are $200 \mathrm{keV}$ of $\mathrm{H}^{+}$and $\mathrm{He}^{+}$, and 400 of $\mathrm{Ar}^{++}$. The $\mathrm{H}_{2} \mathrm{O}_{2} / \mathrm{H}_{2} \mathrm{O}(\%)$ ratio has been plotted as a function of the energy deposited in the target measured in $\mathrm{eV} / 16 \mathrm{amu}$.

the penetration depth of used ions. Their results showed that $\mathrm{H}_{2} \mathrm{O}_{2}$ was produced by all the ions used both at 16 and $77 \mathrm{~K}$. Moreover, they found that the quantity of $\mathrm{H}_{2} \mathrm{O}_{2}$ produced is

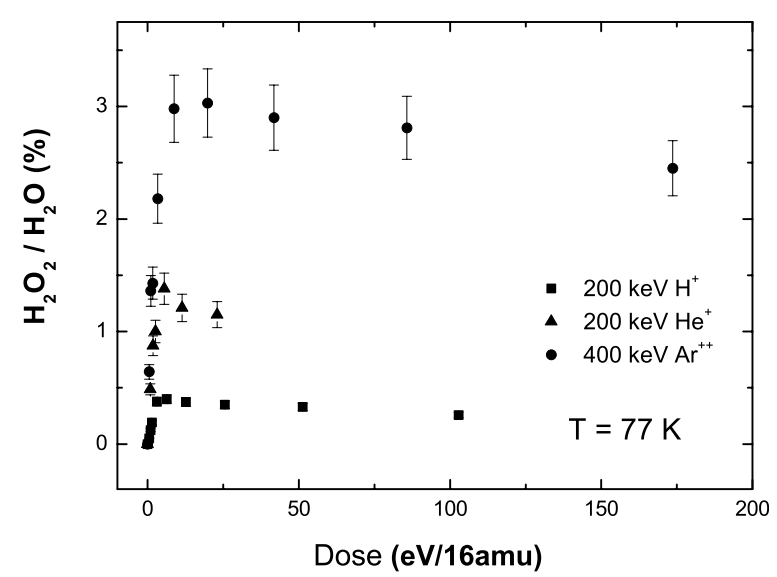

Fig. 5. $\mathrm{H}_{2} \mathrm{O}_{2} / \mathrm{H}_{2} \mathrm{O}(\%)$ production (in number of molecules) after irradiation of thin films of water ice at a temperature of $77 \mathrm{~K}$. The used ions are $200 \mathrm{keV}$ of $\mathrm{H}^{+}$and $\mathrm{He}^{+}$, and 400 of $\mathrm{Ar}^{++}$. The $\mathrm{H}_{2} \mathrm{O}_{2} / \mathrm{H}_{2} \mathrm{O}(\%)$ ratio has been plotted as a function of the energy deposited in the target measured in $\mathrm{eV} / 16 \mathrm{amu}$.

greater for $30 \mathrm{keV} \mathrm{C}^{+}, \mathrm{N}^{+}, \mathrm{O}^{+}$, and $\mathrm{Ar}^{+}$than for $30 \mathrm{keV} \mathrm{H}^{+}$. As the heaviest ions have stopping powers much larger than $\mathrm{H}^{+}$, their conclusion was that the energy released into the sample 
by elastic collisions with target nuclei plays an important role in the process of formation of the hydrogen peroxide molecule. The results obtained in this work reinforce this argument and show that the heaviest ions also form more $\mathrm{H}_{2} \mathrm{O}_{2}$ in thin films.

From Figs. $3-5$ it is clear that the $\mathrm{H}_{2} \mathrm{O}_{2}$ percentage increases quickly as the ion fluence increases. Furthermore, it is observed that the $\mathrm{H}_{2} \mathrm{O}_{2} / \mathrm{H}_{2} \mathrm{O}(\%)$ ratio remains constant or starts to decrease as the fluence keeps growing. The decrease is due to the fact that as the ion fluence increases the water ice column density decreases (water molecules are destroyed and produce radicals that react to form new molecules or are lost as a consequence of sputtering) and therefore there is less available water to form hydrogen peroxide. Moreover, $\mathrm{H}_{2} \mathrm{O}_{2}$ itself is also destroyed by further irradiation.

\section{Discussion}

The number of $\mathrm{H}_{2} \mathrm{O}_{2}$ molecules present in our sample as a function of time is given by the balance between formation and destruction mechanisms (Gomis et al. 2004):

$\frac{\mathrm{d} n_{\mathrm{h}}}{\mathrm{d} t}=\sigma_{\mathrm{f}} \phi n-\sigma_{\mathrm{d}} \phi n_{\mathrm{h}}$

where $n_{\mathrm{h}}$ is the hydrogen peroxide column density; $n$ the $\mathrm{H}_{2} \mathrm{O}$ column density; $\sigma_{\mathrm{f}}$ the $\mathrm{H}_{2} \mathrm{O}_{2}$ formation cross-section; $\sigma_{\mathrm{d}}$ the $\mathrm{H}_{2} \mathrm{O}_{2}$ destruction cross-section and $\phi$ the ion flux.

When the $\mathrm{H}_{2} \mathrm{O}$ column density remains constant a solution for Eq. (2) is the following:

$n_{\mathrm{h}}(t)=n_{\infty}\left(1-\mathrm{e}^{-\sigma_{\mathrm{d}} x}\right)$,

where $x=\phi t$ is the ion fluence and $n_{\infty}$ the asymptotic value for the $\mathrm{H}_{2} \mathrm{O}_{2}$ column density as the ion fluence goes to infinity.

If the $\mathrm{H}_{2} \mathrm{O}$ column density $n$ decreases linearly with ion fluence, a solution for Eq. (2) can be written as follows:

$n_{\mathrm{h}}(t)=\frac{n_{0} \sigma_{\mathrm{f}} \sigma_{\mathrm{d}}+\sigma_{\mathrm{f}} \sigma_{\mathrm{dw}}}{\left(\sigma_{\mathrm{d}}\right)^{2}}\left(1-\mathrm{e}^{-\sigma_{\mathrm{d}} x}\right)-\frac{\sigma_{\mathrm{f}} \sigma_{\mathrm{dw}}}{\sigma_{\mathrm{d}}} x$,

where $n_{0}$ is the initial $\mathrm{H}_{2} \mathrm{O}$ column density and $\sigma_{\mathrm{dw}}$ is $\mathrm{H}_{2} \mathrm{O}$ destruction yield (measured in molecules ion ${ }^{-1}$ ).

We have fitted the $\mathrm{H}_{2} \mathrm{O}_{2}$ column densities as a function of ion fluence using Eqs. (3) and (4). The parameters to be determined by the fit are $\sigma_{\mathrm{d}}$ and $n_{\infty}$ in the case of Eq. (3) and $\sigma_{\mathrm{d}}$ and $\sigma_{\mathrm{f}}$ in the case of Eq. (4). Table 1 shows the values obtained for the hydrogen peroxide destruction and formation cross-sections for each of the used ions at both temperatures.

For the case of $\mathrm{He}^{+}$and $\mathrm{Ar}^{++}$irradiation, $\sigma_{\mathrm{d}}$ and $\sigma_{\mathrm{f}}$ are obtained directly from the nonlinear fit (Eq. (4)). The required values for the initial $\mathrm{H}_{2} \mathrm{O}$ column density $n_{0}$ and the $\mathrm{H}_{2} \mathrm{O}$ destruction yield $\sigma_{\mathrm{dw}}$ are obtained from a linear fit of the points shown in Fig. 1. For those two ions we have used the same values of the water destruction yield for the temperatures of 16 and $77 \mathrm{~K}$.

In contrast, for the case of $\mathrm{H}^{+}$, the $\mathrm{H}_{2} \mathrm{O}$ destruction yield is lower and a constant value for the amount of water ice can be assumed. In this case, the value for $\sigma_{\mathrm{d}}$ has been obtained directly from Eq. (3). The value for $\sigma_{\mathrm{f}}$ has been evaluated from the asymptotic value for the $\mathrm{H}_{2} \mathrm{O}_{2}$ column density $n_{\infty}$ taking into account that $\sigma_{\mathrm{f}} / \sigma_{\mathrm{d}}=n_{\infty} / n$ in which $n$ is the $\mathrm{H}_{2} \mathrm{O}$ column density. The corresponding values for $n_{\infty} \mathrm{ob}-$ tained after fitting the data points with Eq. (3) are $2.1 \times 10^{16}$ and $0.88 \times 10^{16}$ molecules $\mathrm{cm}^{-2}$ at 16 and $77 \mathrm{~K}$ respectively (see Gomis et al. 2004, for further details).

\section{Astrophysical considerations}

Simple molecules are found in the form of ices on silicatic or carbonaceous grain mantles in the circumstellar and interstellar medium. In the formation and evolution of those ices a number of different processes play a role. Actually, those solid molecules can be directly frozen from the gas phase or synthesized on grains via surface reactions or by more energetic processes such as UV photolysis, cosmic ray irradiation and thermal annealing.

To study the role played by each of the latter processes in the evolution of the ice, laboratory experiments which simulate the astrophysical conditions are carried out by different groups. In this way, surface chemistry experiments (Collings et al. 2003; Watanabe \& Kouchi 2002a; Watanabe et al. 2003), UV photolysis (Gerakines et al. 1996; Watanabe \& Kouchi 2002b) and ion irradiation (radiolysis, e.g., Hudson \& Moore 1999, 2000; Palumbo \& Strazzulla 1993; Palumbo et al. 2000; Baragiola et al. 2003) give us information about the basic processes of ice mantle formation, adsorption, diffusion, desorption and reaction pathways.

In the present work we have simulated the low energy cosmic ray radiation expected for ice mantles in the dense ISM. As reported by Greenberg (1982), the time spent by a grain in the interstellar medium is $3 \times 10^{7}-5 \times 10^{8} \mathrm{yr}$. At the densities of dense molecular clouds $\left(10^{3}-10^{5} \mathrm{H}\right.$ atoms $\left.\mathrm{cm}^{-3}\right)$, the timescale for the condensation of molecules on the dust particles is small ( $\sim 10^{5}$ yr; e.g., Tielens \& Allamandola 1987). Thus icy grain mantles suffer irradiation for $10^{5}-5 \times 10^{8} \mathrm{yr}$.

The flux of low energy cosmic rays irradiating grains in the interstellar medium is not well known. A reasonable estimation is a proton flux $J(E=1 \mathrm{MeV})=10 \mathrm{~cm}^{-2} \mathrm{~s}^{-1}$ (Strazzulla \& Johnson 1991). Palumbo \& Strazzulla (1993) estimated a dose released to interstellar ices via cosmic ray bombardment between 0.05 and $50 \mathrm{eV} /$ molecule while Moore (1999) gives values between 3 and $300 \mathrm{eV} /$ molecule assuming a cloud lifetime of $10^{7}$ and $10^{9} \mathrm{yr}$ respectively.

Figure 4 and Table 1 show the maximum value of the $\mathrm{H}_{2} \mathrm{O}_{2} / \mathrm{H}_{2} \mathrm{O}(\%)$ ratio produced after irradiation of thin films of water ice with $\mathrm{H}^{+}, \mathrm{He}^{+}$and $\mathrm{Ar}^{++}$ions at the given energies at $16 \mathrm{~K}$, a temperature that well mimics that of the grains in interstellar dense clouds. We can see that protons produce a maximum ratio of $\sim 1 \%, \mathrm{He}^{+}$of $\sim 2.5 \%$ and for $\mathrm{Ar}^{++}$we measure a value of $\sim 6 \%$. These limits in the production of hydrogen peroxide by ion irradiation put a constraint on the quantity of $\mathrm{H}_{2} \mathrm{O}_{2}$ that can be synthesized on grain mantles in dense molecular clouds. As can be seen from Fig. 4, the levels of deposited energy obtained experimentally cover the range of radiation exposure expected for the ices during the dense cloud lifetimes. Furthermore, the dominant contribution of cosmic rays comes mainly from low energy protons in the $\mathrm{MeV}$ range (Moore 1999), which are comparable to those used in our experiments. 
This justifies the extrapolation of our results to the ISM astrophysical scenario.

To conclude, we wish to stress that our results exclude the possibility that hydrogen peroxide abundances are greater than a few percent of water in interstellar ices. This puts severe limits on the possibility of detecting hydrogen peroxide in interstellar ice mantles. Boudin et al. (1998) derived an upper limit of $6.1 \%$ for the $\mathrm{H}_{2} \mathrm{O}_{2}$ abundance by comparing results obtained from IR spectroscopy experiments on water/hydrogen peroxide mixtures and astronomical observations of the high-mass protostar NGC 7538: IRS 9. Moreover, Smith et al. (2003) have carried out additional laboratory experiments on water/hydrogen peroxide mixtures with subsequent comparison with the spectrum of a low-mass protostar provided by ISO. Smith et al. (2003) stressed the difficulty in unambiguously identifying interstellar hydrogen peroxide ice, although they have not excluded its presence in grain mantles. Further searches for solid $\mathrm{H}_{2} \mathrm{O}_{2}$ require observations with more sensitive instruments.

Acknowledgements. We thank G. A. Baratta and M. E. Palumbo for useful discussions and F. Spinella for his help during laboratory experiments. We are grateful to Dr R. H. Hudson, referee of this paper, for helpful comments and suggestions.

\section{References}

Allamandola, L. J., Sandford, S. A., \& Valero, G. J. 1988, Icarus, 76, 225

Baragiola, R. A., Vidal, R. A., Svendsen, W., et al. 2003, Nucl. Instr. Meth. B, 209, 294

Boogert, A. C. A., \& Ehrenfreund, P. 2004, in Astrophysics of Dust, ed. A. N. Witt, G. C. Clayton, \& B. T. Draine (San Francisco: ASP), ASP Conf. Ser., in press

Boudin, N., Schutte, W. A., \& Greenberg, J. M. 1998, A\&A, 331, 749

Carlson, R. W., Anderson, M. S., Johnson, R. E., et al. 1999, Science, 283, 2062

Collings, M. P., Dever, J. W., Fraser, H. J., McCoustra, M. R. S., \& Williams, D. A. 2003, ApJ, 583, 1058

d'Hendecourt, L. B., Allamandola, L. J., \& Greenberg, J. M. 1985, A\&A, 152, 130

Ehrenfreund, P., \& Fraser, H. 2003, in Solid State Astrochemistry, ed. V. Pirronello, J. Krelowski, \& G. Manicò (Dordrecht: Kluwer), 317

Ehrenfreund, P., \& Schutte, W. 2000, in Astrochemistry: From Molecular Clouds to Planetary Systems, ed. Y. C. Minh, \& E. F. van Dishoeck (San Francisco: ASP), 135

Gerakines, P. A., Schutte, W. A., \& Ehrenfreund, P. 1996, A\&A, 312, 289

Giguere, P. A., \& Harvey, K. B. 1959, J. Molec. Spec., 3, 36

Gomis, O., Satorre, M. A., Strazzulla, G., \& Leto, G. 2004, Planet. Space Sci., 52, 371
Greenberg, J. M. 1982, in Comets, ed. L. L. Wilkening (Tucson: The University of Arizona Press), 131

Hasegawa, T. I., \& Herbst, E. 1993, MNRAS, 261, 83

Hasegawa, T. I., Herbst, E., \& Leung, C. M. 1992, ApJS, 82, 167

Hudson, R. L., \& Moore, M. H. 1999, Icarus, 140, 451

Hudson, R. L., \& Moore, M. H. 2000, A\&A, 357, 787

Hudson, R. L., \& Moore, M. H. 2001, J. Geophys. Res., 106, 33275

Johnson, R. E. 1998, in Solar System Ices, ed. B. Schmitt, B. C. de Bergh, \& M. Festou (Dordrecht: Kluwer), 303

Leto, G., \& Baratta, G. A. 2003, A\&A, 397, 7

Miller, R. L., \& Horning, D. F. 1961, J. Chem. Phys., 34, 265

Moore, M. H. 1999, in Solid Interstellar Matter: the ISO Revolution, ed. L. d'Hendecourt, C. Joblin, \& A. Jones (Berlin: Springer-Verlag), 199

Moore, M. H., \& Hudson, R. L. 2000, Icarus, 145, 282

Moore, M. H., Hudson, R. L., \& Gerakines, P. A. 2001, Spectrochim. Acta A, 57, 843

Palumbo, M. E., Pendleton, Y. J., \& Strazzulla, G. 2000, ApJ, 542, 890

Palumbo, M. E., \& Strazzulla, G. 1993, A\&A, 269, 568

Pendleton, Y. J., \& Chiar, J. E. 1997, in From Stardust to Planetesimals, ed. Y. Pendleton, \& A. G. G. M. Tielens (San Francisco: ASP), ASP Conf. Ser., 122, 179

Sandford, S. A., Allamandola, L. A., \& Bernstein, M. P. 1997, in From Stardust to Planetesimals, ed. Y. Pendleton \& A. G. G. M. Tielens (San Francisco: ASP), ASP Conf. Ser., 122, 201

Schmitt, B., de Bergh, C., \& Festou, M. 1998, Solar System Ices (Dordrecht: Kluwer)

Shalabiea, O. M., \& Greenberg, J. M. 1994, A\&A, 290, 266

Smith, R. G., Charnley, S., Pendleton, Y., et al. 2003, in Astrophysics of Dust, ed. A. N. Witt

Smith, R. G., Sellgren, K., \& Tokunaga, A. T. 1989, ApJ, 344, 413

Strazzulla, G. 1998, in Solar System Ices, ed. B. Schmitt, C. de Bergh, \& M. Festou (Dordrecht: Kluwer), 281

Strazzulla, G., Baratta, G. A., \& Palumbo, M. E. 2001, Spectrochim. Acta A, 57, 825

Strazzulla, G., \& Johnson, R. E. 1991, in Comets in the Post-Halley Era, ed. R. L. Newburn, M. Neugebauer, \& J. Rahe (Dordrecht: Kluwer), 243

Tielens, A. G. G. M., \& Allamandola, L. J. 1987, in Physical Processes in Interstellar Clouds, ed. G. E. Morfill, \& M. Scholer (Dordrecht: Reidel), 333

Tielens, A. G. G. M., \& Hagen, W. 1982, A\&A, 114, 245

Watanabe, N., \& Kouchi, A. 2002a, ApJ, 571, L173

Watanabe, N., \& Kouchi, A. 2002b, ApJ, 567, 651

Watanabe, N., Shiraki, T., \& Kouchi, A. 2003, ApJ, 588, L121

Whittet, D. C. B., \& Tielens, A. G. G. M. 1997, in From Stardust to Planetesimals, ed. Y. Pendleton, \& A. G. G. M. Tielens (San Francisco: ASP), ASP Conf. Ser., 122, 161

Willner, S. P., Gillet, F. C., Herter, T. L., et al. 1982, ApJ, 253, 174

Ziegler, J. F., Biersack, J. P., \& Littmark, U. 1985, The Stopping and Range of Ions in Solids (New York: Pergamon Press) 